\title{
Fondaparinux vs. enoxaparin for the prevention of venous thromboembolism after total hip replacement: A meta-analysis
}

\author{
WEN-JUN DONG, HUI-JUAN QIAN, YAN QIAN, LING ZHOU and SAN-LIAN HU \\ Department of Nursing, Shanghai Jiao Tong University Affiliated Sixth People's Hospital, Shanghai 200233, P.R. China
}

Received November 5, 2015; Accepted May 17, 2016

DOI: 10.3892/etm.2016.3351

\begin{abstract}
The aim of the study was to compare the efficacy and safety profiles of fondaparinux and enoxaparin in preventing the venous thromboembolism (VTE) after total hip replacement. A systematic literature search in the PubMed, EMBASE and Cochrane library databases was performed to identify relevant articles published in English since inception up to November 16, 2014. The efficacy outcomes were all VTE, total deep vein thrombosis (DVT) and symptomatic VTE, while the safety outcome was major bleeding. Four eligible studies were included in the meta-analysis. The incidences of total VTEs and DVTs were significantly decreased with the use of fondaparinux when compared to enoxaparin [total VTE: risk ratio $(\mathrm{RR})=0.59,95 \%$ confidence interval $(\mathrm{CI})$ : 0.46-0.74, $\mathrm{P}<0.01$; total DVT: $\mathrm{RR}=0.58,95 \% \mathrm{CI}$ : 0.46-0.74, $\mathrm{P}<0.01]$. Significantly fewer symptomatic VTEs and major bleeding cases were observed for the enoxaparin group (symptomatic VTE: $\mathrm{RR}=2.62,95 \% \mathrm{CI}: 1.07-6.45, \mathrm{P}=0.04$; major bleeding: $\mathrm{RR}=1.75,95 \% \mathrm{CI}: 1.20-2.53, \mathrm{P}<0.01)$. In conclusion, the pooled analysis revealed that, compared to enoxaparin, fondaparinux was significantly more effective in preventing VTE after total hip replacements in terms of total VTEs and DVTs, although this was accompanied with an increased risk of major bleeding. However, the pooled analysis of two small sample trials from Japan failed to demonstrate any significant advantage for fondaparinux compared to enoxaparin.
\end{abstract}

\section{Introduction}

Fondaparinux is a selective indirect inhibitor of activated factor Xa that has been shown to have a preventive effect on venous thromboembolism (VTE) $(1,2)$. Total hip replacement, also known as arthroplasty, is a relatively common and highly

Correspondence to: San-Lian $\mathrm{Hu}$, Department of Nursing, Shanghai Jiao Tong University Affiliated Sixth People's Hospital, 600 Yishan Road, Shanghai 200233, P.R. China

E-mail: sanlian_hu1@163.com

Key words: venous thromboembolism, fondaparinux, enoxaparin, total hip replacement successful orthopaedic surgery. It has been estimated that, by the year 2030, the number of total hip replacement surgeries in the United States is likely to increase significantly with projected numbers of 572,000 for primary and 96,700 for revision patterns (3). Postoperative VTE, comprising deep vein thrombosis (DVT) and pulmonary embolism, is the most common post-operation complications following total hip replacement. These complications are widely linked to substantial short/long-term morbidity, mortality and medical costs (4-6). Considering the dangers associated with VTE, thromboprophylaxis with effective and safe anticoagulation in patients undergoing total hip replacements is of critical significance.

Low-molecular-weight heparins such as enoxaparin were shown to be effective and safe for postoperative VTE prevention after total hip replacement surgeries (7). Enoxaparin inhibits the factor IIa (thrombin) and factor Xa activities, indirectly, via antithrombin activation (8). Unlike enoxaparin, fondaparinux, a synthetic pentasaccharide, is an indirect selective, and reversible Xa inhibitor factor. Fondaparinux is the first member of a newly introduced class of synthetic antithrombotic agents that has been studied for their preventive effects on VTE in patients undergoing orthopaedic surgeries $(9,10)$.

Inconsistent results were reported from two large randomized controlled trials (RCTs) that were conducted to compare the efficacy and the safety issues related to fondaparinux and enoxaparin usage in thromboprophylaxis cases $(11,12)$. Two other trials were completed in Japan on the same subject (the use of the two agents in thromboprophylaxis following total hip replacement) $(13,14)$. However, to the best of our knowledge, no other meta-analysis exclusively investigated this subject. Therefore, the current meta-analysis using available data was conducted to better elucidate the efficacy and safety of fondaparinux compared to enoxaparin for the prevention of VTE after total hip replacement surgery.

\section{Materials and methods}

Literature search. A systematic literature search in the PubMed, EMBASE and Cochrane library databases was performed to identify relevant articles published in English language since inception up to November 16, 2014. The main key words used for this search were fondaparinux, enoxaparin and hip replacement. In addition, reference lists of eligible articles were screened for additional articles. 


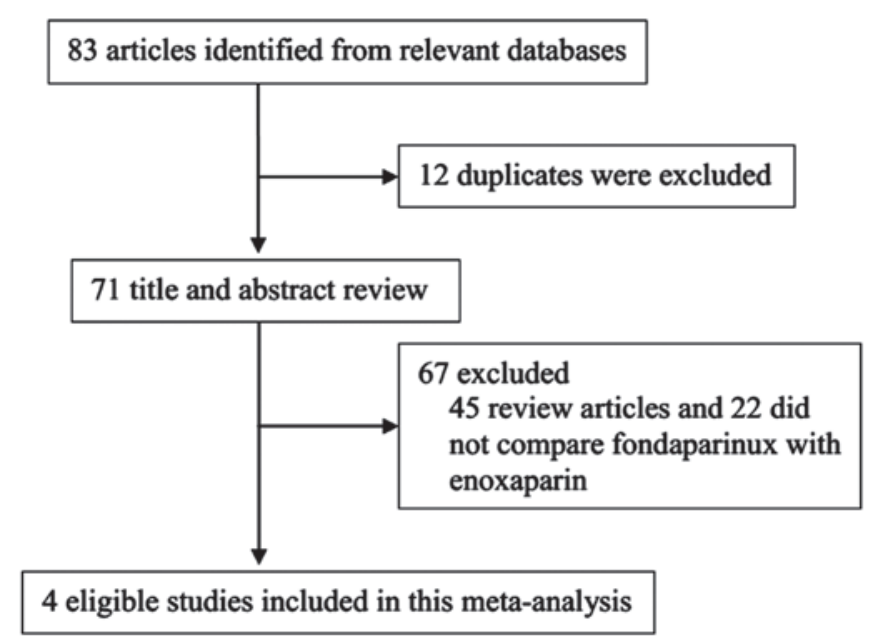

Figure 1. Process of the study selection.

Study selection and data extraction. Published studies were included if they met the following inclusion criteria: i) fondaparinux was compared to enoxaparin in preventing VTE after total hip replacement; and ii) sufficient information regarding the efficacy and safety outcome was available. Studies without any reference to the comparative assessment of the efficacy and safety of fondaparinux and enoxaparin in total hip replacement were excluded. For those studies producing multiple publications with the same population we only included the most recent report, and for the studies with the same results published in different journals we selected the most complete report. Retrospective studies, reviews, animal studies, and studies lacking sufficient data were excluded.

In accordance with the preferred reporting items for systematic reviews and meta-analyses statement (15), two investigators independently extracted the following data from each study: name of first author, publication year, sample size, relevant data about treatment and follow-up. The risk of bias assessment of included studies was evaluated by the two investigators using the Cochrane risk of bias assessment tool (16). Any discrepancies were resolved through discussion with a third investigator.

Statistical analysis. The primary efficacy outcomes used in this study were VTE, total DVT and symptomatic VTE. The primary safety outcome used was major bleeding, defined as in the included trials. Risk ratios (RRs) with their 95\% confidence intervals (CIs) were calculated using the random effects or fixed effects model depending on the level of heterogeneity. The heterogeneity across studies was examined by the Cochran's Q and $\mathrm{I}^{2}$ statistics (17). The fixed effects model was selected for the homogenous outcomes $\left(\mathrm{P} \geq 0.05\right.$ and $\left.\mathrm{I}^{2}<50 \%\right)$ and the random effects model was applied for heterogeneous outcomes $\left(\mathrm{P}<0.05\right.$ or $\left.\mathrm{I}^{2} \geq 50 \%\right)$. $\mathrm{P}<0.05$ was considered to indicate a statistically significant difference. Data analyses were performed using the Review Manger 5.2 version software (Cochrane Library Software, Oxford, UK).

\section{Results}

Search results. The process of study selection is shown in Fig. 1. A total of 83 articles were potentially relevant to our search term. Of the 83 articles, 12 were excluded since they were duplicate publications. After screening the titles and abstracts, another 67 articles were excluded as they were not in accordance with the inclusion criteria. No additional eligible articles were identified via screening the reference lists of identified primary studies. Consequently, 4 articles published between 2002 and 2014 with 5,164 patients were included in the present meta-analysis (11-14).

Characteristics of included studies. The main characteristics of the chosen studies (type of study design, study area, characteristics of the included population, treatment protocol, number of patients and follow ups) are shown in Table I. Three studies were double-blind RCTs with a randomized total population of 4,755 patients (11-13) and the remaining one was a multicenter prospective cohort study with 409 patients who received fondaparinux or enoxaparin following total hip replacement (14). The included RCTs were conducted exclusively on adult patients undergoing total hip replacement surgeries, while the cohort study of Migita et al (14) involved the patients undergoing total knee replacement, which was not a subject of interest for our meta-analysis.

The first injection of $2.5 \mathrm{mg}$ fondaparinux was administered either 4-8 h or 16-20 h after the surgery in three RCTs, while the patients in the cohort study received their first injection $24 \mathrm{~h}$ after the wound closure. The injected doses were based on patient body masses (patients weighing $<50 \mathrm{~kg}$ received a single daily subcutaneous injection of $1.5 \mathrm{mg}$ and those weighing $\geq 50 \mathrm{~kg}$ received an injection of $2.5 \mathrm{mg}$ fondaparinux in the same manner). Enoxaparin dose and regimen in the trials varied based on different approval standards. The results of quality assessment are shown in Fig. 2. Only 2 large RCTs had a low risk of bias in all the assessment factors $(11,12)$, while the 2 studies from Japan only had a low risk of bias regarding attrition and reporting, with unclear or high risk of bias in other aspects $(13,14)$.

The efficacy outcomes including VTE, total DVT and symptomatic VTE were reported in the 4 trials and a fixed effects model was used to pool the data due to relatively homogeneity across the studies. The pooled analysis revealed that, compared with enoxaparin, the risk of VTE and total DVT 


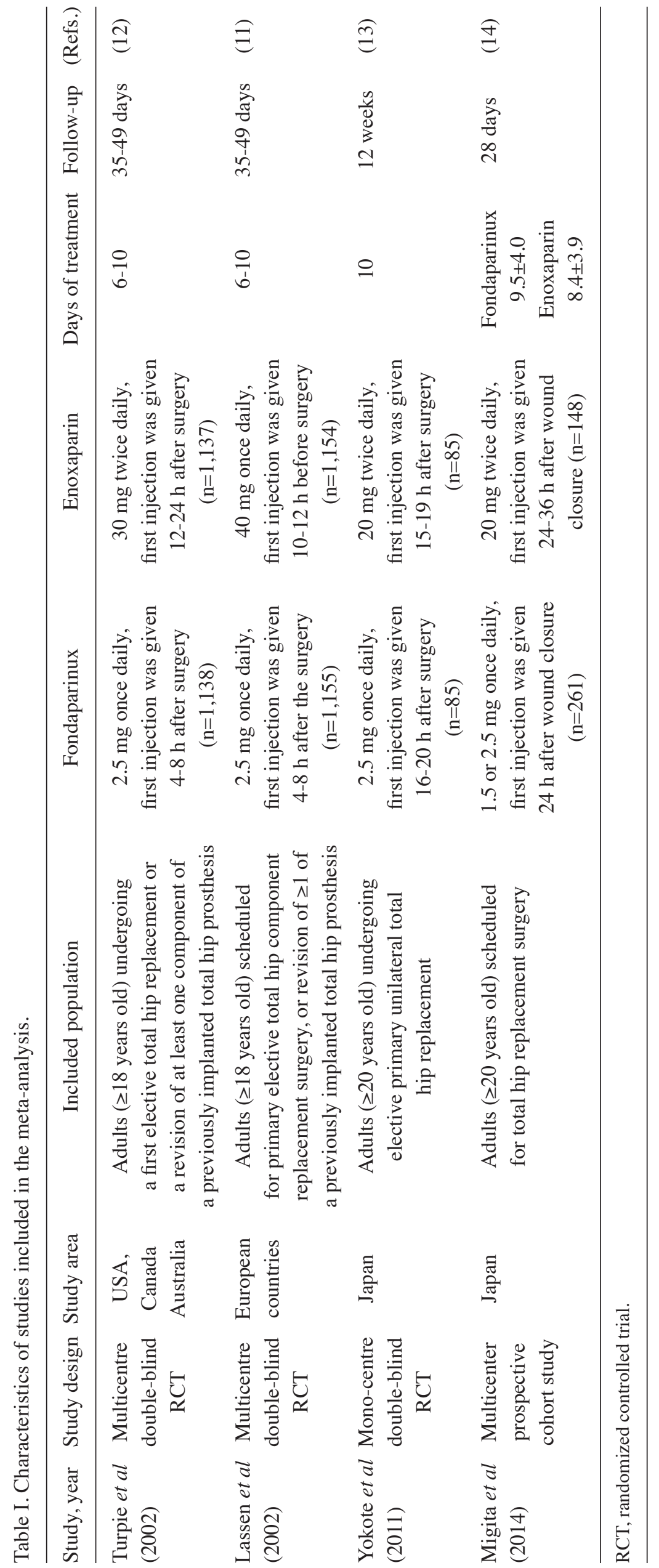



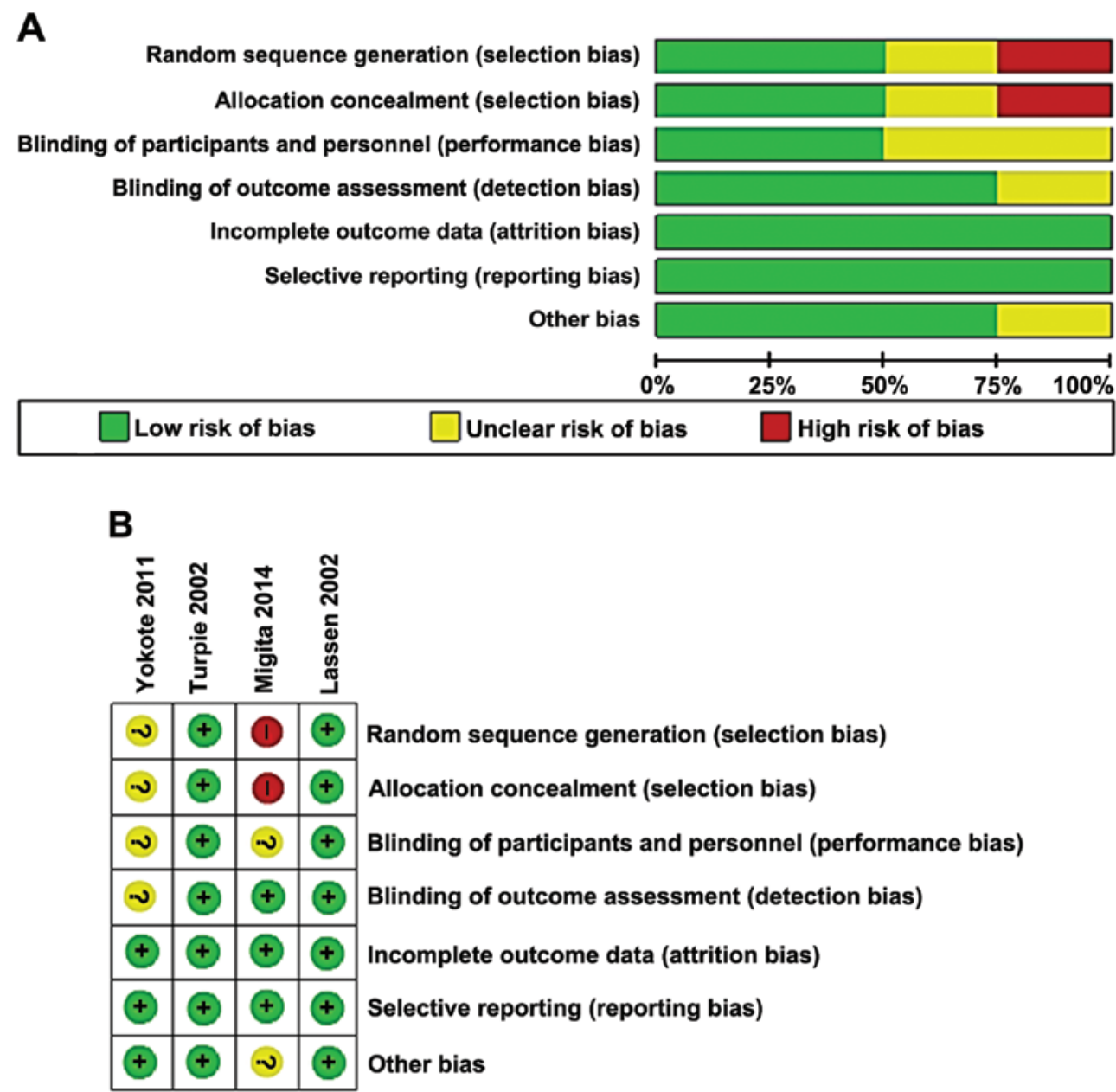

Figure 2. Assessment of the risk of bias in the included studies. (A) Methodological quality graph: authors' judgment about each methodological quality item presented as percentages across the included studies; (B) Methodological quality summary: authors' judgment about each methodological quality item for each included study. +, low risk of bias; ?, unclear risk of bias; -, high risk of bias.

associated with the fondaparinux application was significantly lower (all VTE: $\mathrm{RR}=0.59,95 \% \mathrm{CI}$ : 0.46-0.74, $\mathrm{P}<0.01$; $\mathrm{P}_{\text {heterogeneity }}=0.15, \mathrm{I}^{2}=43 \%$; total DVT: $\mathrm{RR}=0.58,95 \% \mathrm{CI}$ : 0.46-0.74, $\mathrm{P}<0.01 ; \mathrm{P}_{\text {heterogeneity }}=0.18, \mathrm{I}^{2}=39 \%$ ) (Fig. 3). Slightly fewer symptomatic VTE was found in the enoxaparin group as compared to the fondaparinux group $(\mathrm{RR}=2.62,95 \% \mathrm{CI}$ : 1.07-6.45, $\mathrm{P}=0.04 ; \mathrm{P}_{\text {heterogeneity }}=0.20, \mathrm{I}^{2}=35 \%$ ) (Fig. 3).

Safety outcome. Major bleeding was considered to be the primary safety outcome in the 4 trials. Notably, we used more reliable data from the prospective cohort study that provided the data on major bleeding cases after adjustment for baseline risk differences (each of the two treatments have 144 patients). A fixed effects model was used to pool data and the results showed a favorable outcome for the enoxaparin group as compared to the fondaparinux group $(\mathrm{RR}=1.75,95 \% \mathrm{CI}$ : 1.20-2.53, $\mathrm{P}<0.01 ; \mathrm{P}_{\text {heterogeneity }}=0.23, \mathrm{I}^{2}=32 \%$ ) (Fig. 3).

Sensitivity analysis. Although there was no significant heterogeneity across these studies, the methodological differences may have caused some potential bias that influenced the pooled results. Sensitivity analysis was conducted to assess the stability of the results by removing the two lower quality studies from Japan one at a time. Table II shows the results of the sensitivity analysis. Although larger 95\% CIs were observed, following removal of the Migita et al (14) study, results were in accordance with the initial pooled results obtained from the 4 trials. After removing the two lower quality studies, favorable results were present for fondaparinux with regard to VTE and total DVT. No significant differences were identified between the two treatments concerning the symptomatic VTE with a relatively large $95 \%$ CI. Of note, there was significant heterogeneity between the 2 large RCTs with regard to efficacy outcome. In contrast to the significant results mentioned above, the pooled analysis of 2 trials from Japan failed to demonstrate any significant advantages, in terms of efficacy and safety outcomes, for fondaparinux application compare to enoxaparin (data not shown).

\section{Discussion}

The present meta-analysis of 4 trials ( 3 RCTs and a prospective cohort study) was conducted to evaluate the efficacy and safety profiles of fondaparinux and enoxaparin for thromboprophylaxis after total hip replacement surgeries. Relative homogeneity across the 4 studies was detected and the fixed effects model was used to pool the data. The pooled results showed that, in elective total hip replacements, fondaparinux 
Table II. Results of sensitivity analysis.

\begin{tabular}{|c|c|c|c|c|c|c|}
\hline $\begin{array}{l}\text { Omitting } \\
\text { study }\end{array}$ & $\begin{array}{l}\text { Included } \\
\text { study }\end{array}$ & All VTE & Total DVT & $\begin{array}{l}\text { Symptomatic } \\
\text { VTE }\end{array}$ & $\begin{array}{l}\text { Major } \\
\text { bleeding }\end{array}$ & (Refs.) \\
\hline Migita et al (2014) & 3 & $\begin{array}{c}\mathrm{RR}=0.63, \\
95 \% \mathrm{CI}: 0.39-1.01 \\
\mathrm{P}=0.07, \mathrm{I}^{2} \%=62 \%\end{array}$ & $\begin{array}{c}\mathrm{RR}=0.62 \\
95 \% \mathrm{CI}: 0.39-0.99 \\
\mathrm{P}=0.09, \mathrm{I}^{2} \%=59 \%\end{array}$ & $\begin{array}{c}\mathrm{RR}=3.66 \\
95 \% \mathrm{CI}: 1.29-10.34 \\
\mathrm{P}=0.35, \mathrm{I}^{2} \%=6 \%\end{array}$ & $\begin{array}{c}\mathrm{RR}=1.59 \\
95 \% \mathrm{CI}: 1.09-2.32, \\
\mathrm{P}=0.48, \mathrm{I}^{2} \%=0 \%\end{array}$ & (14) \\
\hline $\begin{array}{l}\text { Yokote et al (2011) } \\
\text { and Migita et al (2014) }\end{array}$ & 2 & $\begin{array}{c}\mathrm{RR}=0.57 \\
95 \% \mathrm{CI}: 0.35-0.95 \\
\mathrm{P}=0.05, \mathrm{I}^{2} \%=74 \%\end{array}$ & $\begin{array}{c}\mathrm{RR}=0.56 \\
95 \% \mathrm{CI}: 0.35-0.92 \\
\mathrm{P}=0.07, \mathrm{I}^{2} \%=70 \%\end{array}$ & $\begin{array}{c}\mathrm{RR}=3.52 \\
95 \% \mathrm{CI}: 0.58-21.32 \\
\mathrm{P}=0.14, \mathrm{I}^{2} \%=53 \%\end{array}$ & $\begin{array}{c}\mathrm{RR}=1.59 \\
95 \% \mathrm{CI}: 1.09-2.32, \\
\mathrm{P}=0.48, \mathrm{I}^{2} \%=0 \%\end{array}$ & $(13,14)$ \\
\hline
\end{tabular}

VTE, venous thromboembolism; DVT, deep vein thrombosis; RR, risk ratio; CI, confidence interval; $\mathrm{P}, \mathrm{P}_{\text {heterogeneity }}$.

A

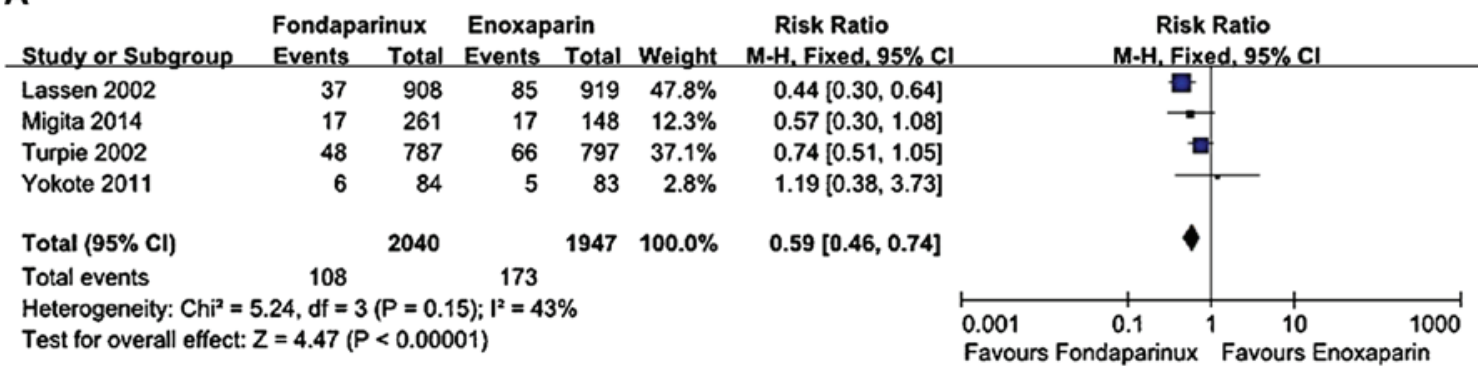

B

Fondaparinux Enoxaparin Risk Ratio

Study or Subgroup Events Total Events Total Weight M-H, Fixed, 95\% C

$\begin{array}{lllllll}\text { Lassen } 2002 & 36 & 908 & 83 & 918 & 47.9 \% & 0.44[0.30,0.64]\end{array}$

$\begin{array}{lllllll}\text { Migita 2014 } & 17 & 261 & 17 & 148 & 12.6 \% & 0.57[0.30,1.08]\end{array}$

$\begin{array}{lllllll}\text { Turpie 2002 } & 44 & 748 & 65 & 796 & 36.6 \% & 0.72[0.50,1.04]\end{array}$

$\begin{array}{lllllll}\text { Yokote } 2011 & 6 & 84 & 5 & 83 & 2.9 \% & 1.19[0.38,3.73]\end{array}$

Total $(95 \% \mathrm{Cl})$

$2001 \quad 1945 \quad 100.0 \% \quad 0.58[0.46,0.74]$

Total events $103 \quad 170$

Heterogeneity: $\mathrm{Chi}^{2}=4.90, \mathrm{df}=3(P=0.18) ; I^{2}=39 \%$

Test for overall effect: $Z=4.50(P<0.00001)$

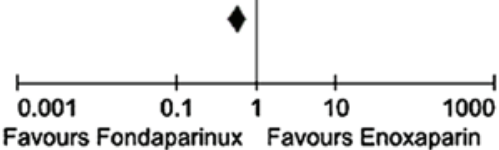

C

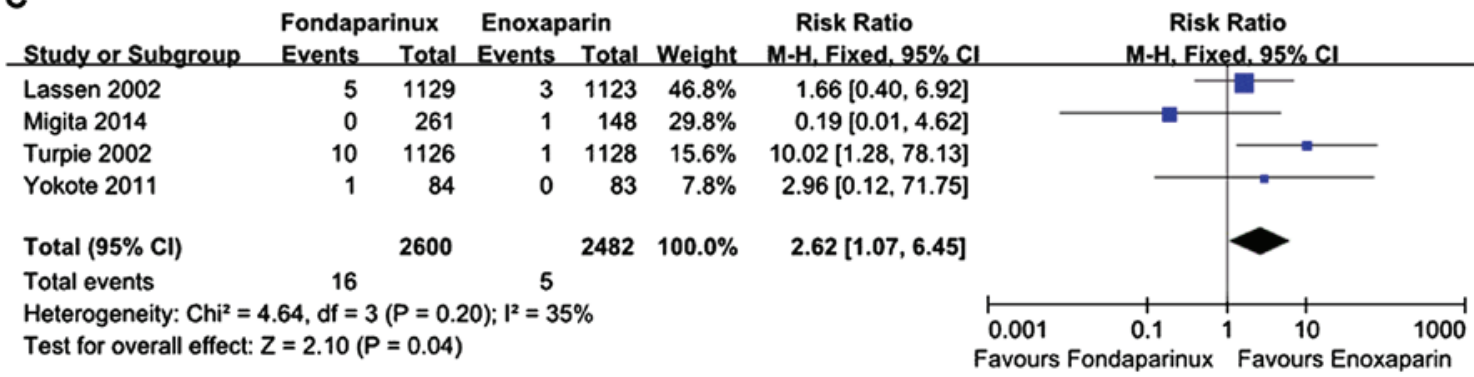

D

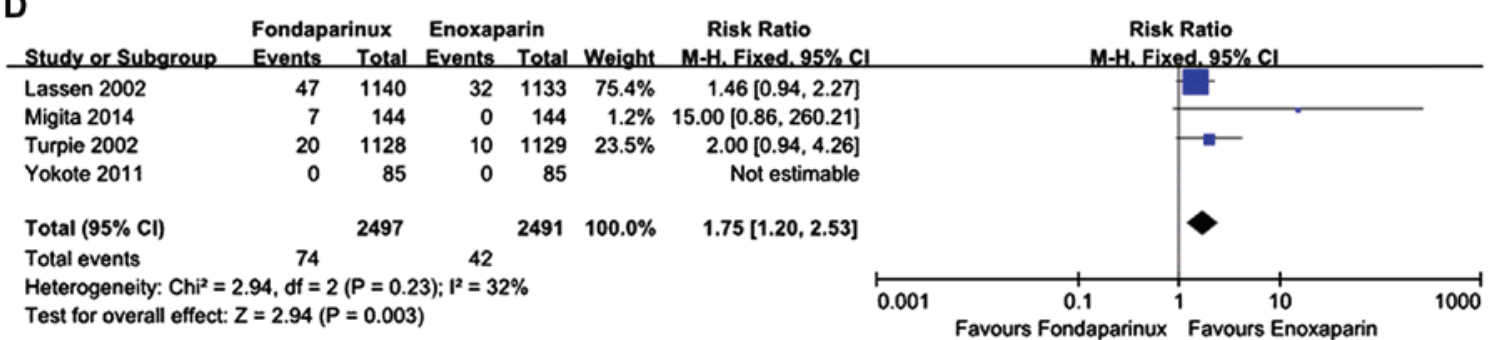

Figure 3. Forest plots of efficacy outcomes and safety outcome (major bleeding) between the 2 treatments. Risk of (A) venous thromboembolism (VTE); (B) total deep vein thrombosis; (C) symptomatic VTE; and (D) major bleeding. CI, confidence interval. 
achieved an approximately $40 \%$ overall reduction in the risk of total VTE and DVT when compared to enoxaparin. An approximately $60 \%$ increased risk of major bleeding was found when fondaparinux was applied. Notably, sensitivity analysis linked to the 2 large RCTs also confirmed this finding although larger 95\% CIs were observed. However, pooled analysis of 2 small sample trials from Japan failed to demonstrate any significant advantages for fondaparinux $(13,14)$.

The observed favorable efficacy profile associated with fondaparinux in the present study is consistent with that of the previous meta-analysis conducted on 4 RCTs in which superior efficacy of fondaparinux over enoxaparin was demonstrated with a $45.3 \%$ reduction in risk of total VTE in total hip replacements (18). Nevertheless, the reduction achieved by fondaparinux was 56 and $26 \%$ in the study that assessed the efficacy and safety of a once-daily regimen of $2.5 \mathrm{mg}$ fondaparinux compared to a once-daily regimen of $40 \mathrm{mg}$ enoxaparin, and a twice-daily regimen of $30 \mathrm{mg}$ enoxaparin, respectively $(11,12)$. The superior efficacy of fondaparinux may be linked to the unique action mechanism of fondaparinux, and its highly reproducible and predictable linear pharmacokinetics (9). The difference observed in results of the reduced risk between 2 RCTs may be attributed to the treatment schedule of enoxaparin. However, no significant difference in efficacy outcomes was identified between the two treatments as demonstrated by the 2 trials from Japan $(13,14)$. The genetic differences are responsible for the differences observed between the 2 large RCTs and 2 trials from Japan. Studies have shown that in Asia thrombophilias are predominantly attributed to the natural anti-coagulants deficiencies (protein $\mathrm{C}$, protein $\mathrm{S}$, and antithrombin), whereas factor $\mathrm{V}$ Leiden and prothrombin G20210A gene mutation are rarely reported (19).

Major bleeding is considered the primary safety concern with the use of anticoagulants in patients undergoing major orthopedic surgery (7). In the current study, a significant increased risk of major bleeding was found for fondaparinux. However, no significant differences were observed between the two treatments concerning the risk of major bleeding as indicated by a separate trial. We took into account that the different definitions for major bleeding may create some bias across the studies that would have influenced the pooled results.

Accordingly, the results presented in this study should be interpreted with caution, considering the limitations. First, we did not evaluate the publication bias since the eligible studies were limited and a practical assessment could not be carried out. Thus, the potential publication bias should be considered regarding the interpretation of the result. Second, we only evaluated the primary efficacy/safety outcomes including VTE, total DVT, symptomatic VTE and major bleeding. Secondary outcomes such as all-cause mortality, clinically relevant non-major bleeding and liver function were not evaluated.

In conclusion, the pooled analysis revealed that fondaparinux had a superior efficacy compared to enoxaparin in the prevention of VTE after total hip replacement in terms of total VTE and DVT. Nonetheless it increased the risk of major bleeding. Therefore, a well-designed RCT performed on a specific population is required.

\section{References}

1. Eikelboom JW, Karthikeyan G, Fagel N and Hirsh J: American Association of Orthopedic Surgeons and American College of Chest Physicians guidelines for venous thromboembolism prevention in hip and knee arthroplasty differ: what are the implications for clinicians and patients? Chest 135: 513-520, 2009.

2. Lee S, Hwang JI, Kim Y, Yoon PW, Ahn J and Yoo JJ: Venous thromboembolism following hip and knee replacement arthroplasty in Korea: a nationwide study based on claims registry. $\mathbf{J}$ Korean Med Sci 31: 80-88, 2016.

3. Kurtz S, Ong K, Lau E, Mowat F and Halpern M: Projections of primary and revision hip and knee arthroplasty in the United States from 2005 to 2030. J Bone Joint Surg Am 89: 780-785, 2007.

4. Selby R and Geerts W: Prevention of venous thromboembolism: Consensus, controversies, and challenges. Hematology (Am Soc Hematol Educ Program) 2009: 286-292, 2009.

5. Paolucci F, Clavies MC, Donat F and Necciari J: Fondaparinux sodium mechanism of action: Identification of specific binding to purified and human plasma-derived proteins. Clin Pharmacokinet 41 (Suppl 2): 11-18, 2002.

6. Keam SJ and Goa KL: Fondaparinux sodium. Drugs 62: 1673-1685, 1686-1687, 2002.

7. Geerts WH, Heit JA, Clagett GP, Pineo GF, Colwell CW, Anderson FA and Wheeler HB: Prevention of venous thromboembolism. Chest 119 (Suppl 1): 132S-175S, 2001

8. Ibbotson T and Goa KL: Enoxaparin: An update of its clinical use in the management of acute coronary syndromes. Drugs 62: 1407-1430, 2002.

9. Turpie AG, Eriksson BI, Lassen MR and Bauer KA: Fondaparinux, the first selective factor Xa inhibitor. Curr Opin Hematol 10: 327-332, 2003.

10. Bergqvist D: Review of fondaparinux sodium injection for the prevention of venous thromboembolism in patients undergoing surgery. Vasc Health Risk Manag 2: 365-370, 2006.

11. Lassen MR, Bauer KA, Eriksson BI and Turpie AG; European Pentasaccharide Elective Surgery Study (EPHESUS) Steering Committee: Postoperative fondaparinux versus preoperative enoxaparin for prevention of venous thromboembolism in elective hip-replacement surgery: a randomised double-blind comparison. Lancet 359: 1715-1720, 2002.

12. Turpie AG, Bauer KA, Eriksson BI and Lassen MR; PENTATHALON2000 Study Steering Committee: Postoperative fondaparinux versus postoperative enoxaparin for prevention of venous thromboembolism after elective hip-replacement surgery: a randomised double-blind trial. Lancet 359: 1721-1726, 2002.

13. Yokote R, Matsubara M, Hirasawa N, Hagio S, Ishii K and Takata C: Is routine chemical thromboprophylaxis after total hip replacement really necessary in a Japanese population? J Bone Joint Surg Br 93: 251-256, 2011.

14. Migita K, Bito S, Nakamura M, Miyata S, Saito M, Kakizaki H, Nakayama Y, Matsusita T, Furuichi I, Sasazaki Y, et al: Venous thromboembolism after total joint arthroplasty: results from a Japanese multicenter cohort study. Arthritis Res Ther 16: R154, 2014.

15. Liberati A, Altman DG, Tetzlaff J, Mulrow C, Gøtzsche PC, Ioannidis JP, Clarke M, Devereaux PJ, Kleijnen J and Moher D: The PRISMA statement for reporting systematic reviews and meta-analyses of studies that evaluate health care interventions: explanation and elaboration. Ann Intern Med 151: W65-94, 2009.

16. Higgins JPT and Green S (eds): Cochrane Handbook for Systematic Reviews of Interventions Version 5.1.0 (updated March 2011). The Cochrane Collaboration 2011. Available from www.cochrane-handbook.org.

17. Higgins JP, Thompson SG, Deeks JJ and Altman DG: Measuring inconsistency in meta-analyses. BMJ 327: 557-560, 2003.

18. Turpie AG, Bauer KA, Eriksson BI and Lassen MR: Fondaparinux vs enoxaparin for the prevention of venous thromboembolism in major orthopedic surgery: a meta-analysis of 4 randomized double-blind studies. Arch Intern Med 162: 1833-1840, 2002.

19. Angchaisuksiri P: Venous thromboembolism in Asia - an unrecognised and under-treated problem? Thromb Haemost 106: 585-590, 2011. 